\title{
Research advances of the drift reducing technologies in application of agricultural aviation spraying
}

\author{
Shengde Chen ${ }^{1,2}$, Yubin Lan ${ }^{1,2 *}$, Zhiyan Zhou ${ }^{2,3}$, Xiaoling Deng ${ }^{1,2}$, Juan Wang ${ }^{4}$ \\ (1. College of Electronic Engineering, South China Agricultural University, Guangzhou 510642, China; \\ 2. National Center for International Collaboration Research on Precision Agricultural Aviation Pesticides Spraying Technology, \\ Guangzhou 510642, China; \\ 3. College of Engineering, South China Agricultural University, Guangzhou 510642, China; \\ 4. Mechanical and Electrical Engineering College, Hainan University, Haikou 570228, China)
}

\begin{abstract}
With the gradual deterioration of the ecological environment and the increase in requirements for the quality of modern life, the use of pesticides is bound to develop towards higher pesticide utilization and less environmental pollution, and the low-volume spraying for agricultural aviation operation combined with the Drift Reducing Technologies (DRTs) may be a useful way to achieve this goal. Based on an analysis of the spray drift mechanism and the primary factors influencing aerial spraying, previous research on DRTs in aerial spraying were reviewed and summarized, and it was found that DRTs in aerial spraying can effectively reduce the environmental pollution caused by pesticide drift by reducing the spraying amount of pesticides and improving the control effect of pesticides, included aerial electrostatic spray technology, aerial spray adjuvant, aerial air-assisted spray technology, drift reducing nozzles and aerial variable-rate spray technology. And according to the analysis of the current research status, some suggestions and countermeasures to reduce droplet drift of agricultural aviation spraying were put forward from the aspects of strengthening the research on DRTs for plant protection Unmanned Aerial Vehicle (UAV) and adopting reasonable DRTs methods. It is hoped that provide reference and guidance for the enterprises' product improvement and users' practical operation, and play the advantages of precision agricultural aviation spraying fully.
\end{abstract}

Keywords: agricultural aviation spraying, pesticide, drift, DRTs, precision agriculture

DOI: $10.25165 /$ j.ijabe.20211405.6225

Citation: Chen S D, Lan Y B, Zhou Z Y, Deng X L, Wang J. Research advances of the drift reducing technologies in application of agricultural aviation spraying. Int J Agric \& Biol Eng, 2021; 14(5): 1-10.

\section{Introduction}

The invention and use of pesticides are keys for increasing crop yield, which is the main purpose of agricultural production. It is estimated that agrochemicals prevent up to $45 \%$ of the loss of the world's food supply ${ }^{[1,2]}$. However, there are continued concerns by both national governments and the general public surrounding the potential adverse effects of pesticides on the improvement of modern living quality. Of most concern is the impact on the environment and risks to human health during application in the field and from residues remaining in the food chain $^{[3]}$. For example, the 1.3 million $t$ of pesticides used in China each year, are believed to contaminate 10 billion $\mathrm{hm}^{2}$ of the cultivated $\operatorname{area}^{[4]}$. As a result, the Ministry of Agriculture and Rural Affairs of China passed a reduction program for chemical fertilizers and pesticides, requiring a net-zero growth in chemical

Received date: 2020-10-19 Accepted date: 2021-01-29

Biographies: Shengde Chen, $\mathrm{PhD}$, research associate, research interest: precision agriculture aviation technology and equipment, Email: shengde-chen@ scau.edu.cn; Zhiyan Zhou, PhD, Professor, research interest: precision agriculture aviation application, Email: zyzhou@scau.edu.cn; Xiaoling Deng, $\mathrm{PhD}$, associate professor, research interest: precision agriculture aviation application, Email: xl-deng@scau.edu.cn; Juan Wang, PhD, associate professor, research interest: precision agriculture aviation application, Email: wj-jdxy@ hainanu.edu.cn.

*Corresponding author: Yubin Lan, $\mathrm{PhD}$, Professor, research interest: precision agricultural aviation application. Mailing address: International Laboratory of Agricultural Aviation Pesticide Spraying Technology (ILAAPST), South China Agricultural University, Guangzhou 510642, China. Tel: +86-20-85281421, Email: ylan@scau.edu.cn. fertilizer and pesticide applications by 2020

As an important part of modern agriculture and one of the important signs reflecting the level of agricultural modernization, the aerial spraying operation has advantages over traditional spraying methods and has gradually become the preferred method for plant protection operations in China ${ }^{[5]}$. Since aerial spraying can now be conducted using unmanned aerial vehicles (UAVs), it is efficient and allows for a quick response to sudden pest outbreaks of pests ${ }^{[6]}$. Labor costs for operation are also low, and crops and the physical structure of soil are not damaged from equipment ${ }^{[7,8]}$. Furthermore, aerial spraying can reduce pesticide application by $15 \%-20 \%$ by using a low or ultra-low volume of spray, and it can be used as an important technical support for a reduction program for chemical fertilizers and pesticides ${ }^{[9]}$.

In an ideal aerial spraying scenario, all pesticides would be deposited on target pests or crops in the target area ${ }^{[10]}$. However, flight speed and flight height of aerial spraying, and the existence of the turbulent wind field below the rotor can cause droplets to drift. Wang et al. ${ }^{[1]}$ conducted an aerial spraying test using a single-rotor plant protection UAV and found that the cumulative drift rate of droplets reached $14.3 \%-75.8 \%$, with a crosswind wind speed of $0.76-5.50 \mathrm{~m} / \mathrm{s}$. Drift, which makes its way into the non-target environment, is not only a waste of pesticides but can cause problems such as phytotoxicity to sensitive crops, poisoning of humans and animals, and environmental pollution ${ }^{[12,13]}$. Therefore, the issue of drift has become a bottleneck for the application and promotion of aerial spraying.

In order to break through the bottleneck of aerial spraying, low-volume spraying application in combination with DRTs may 
be able to address this challenge. Based on an analysis of spray drift mechanism and the primary factors governing aerial spraying, the research status and progress of DRTs in the application of aerial spraying were reviewed and summarized in this article, including electrostatic spraying technology, spray adjuvant, air-assisted spraying technology, drift reducing nozzles and variable-rate spraying technology, all for aerial applications. Additionally, suggestions and countermeasures were put forth to reduce drift in plant protection UAVs and encourage the adoption of reasonable DRTs. It is hoped that DRTs can effectively reduce the environmental pollution caused by pesticide drift, and play the advantages of precision agricultural aviation spraying fully.

\section{Analysis of spray drift mechanism and influencing} factors

\subsection{Spray drift mechanism}

Spray drift refers to the physical movement of pesticide droplets in the atmosphere from the target area to the non-target area during or after spraying ${ }^{[1]}$. Spray drift is an important problem that cannot be ignored in the process of spraying with the development of ecological agriculture and the improvement of environmental protection requirements. How to reduce spraying drift is always the most important difficulty and hot spot in the field of pesticide application ${ }^{[14]}$.

During the stage of droplet generation, there are two primary causes of droplet drift. First, the liquid pesticide is pushed through the nozzle at a high speed, quickly breaking into droplets upon discharge. The region where droplets are created strongly interacts with the atmosphere, and as a result, the surrounding atmosphere becomes involved in the movement by these high-speed moving droplets when droplets are sprayed into the air, and a turbulent flow field is created. As the momentum between the droplets and the air is constantly changing, the turbulent flow field becomes increasingly stronger and affects the effective deposition of the fine droplets ${ }^{[15]}$. Secondly, a lateral flow field is created by the relative movement of the aerial spraying equipment and the atmosphere, also affecting droplets deposition with the environmental wind field together. When either occurs, droplets are not deposited directly on the target crop, but drift and deposit to the non-target area.

To understand the drift mechanism of droplets, it is necessary to understand the forces that affect droplets in the air. For discrete droplets traveling in a continuous fluid medium, the acting forces on the droplet that affect the droplet acceleration are due to a difference in velocity between the droplet and the fluid and due to the displacement of the fluid particle. The force analysis of droplets during the deposition process is very complicated because droplets are subject to a variety of forces. In order to highlight the action characteristics of the main forces and facilitate people to understand the mathematical model of droplet movement, it was assumed that a single droplet is a spherical particle, and the evaporation of droplets is neglected. All the other forces were disregarded except for gravity, drag, and buoyancy forces. According to Newton's equation of motion, the dynamic model of droplets in a fluid medium is as follows ${ }^{[16]}$ :

$$
m_{d} \frac{d v_{d i}}{d t}=\frac{1}{8} \pi \rho d^{2} c_{d}\left|V_{f}-V_{d i}\right|\left(V_{f}-V_{d i}\right)+\frac{1}{6} \pi d^{3}\left(\rho_{d}-\rho\right) g
$$

where, $m_{d}$ is the droplet mass, $\mathrm{kg}$; $d$ is the droplet diameter, $\mathrm{m}$; suffix $i$ represents the direction $\left(i=x, y\right.$ or $z$ ); $V_{f}$ is the continuous fluid velocity including a turbulent component that implements the turbulent dispersion, $\mathrm{m} / \mathrm{s} ; V_{d i}$ is the discrete droplet velocity, $\mathrm{m} / \mathrm{s}$;
$C_{d}$ represents the drag coefficient; $\rho_{d}$ is the discrete droplet density, $\mathrm{kg} / \mathrm{m}^{3} ; \rho$ is the air density, $\mathrm{kg} / \mathrm{m}^{3} ; g$ is the gravitational acceleration, $\mathrm{m} / \mathrm{s}^{2} ; t$ is the time, $\mathrm{s}$.

Assuming that the $Y$-direction is the downward direction of gravity and the $Z$-direction is the direction of airflow generated by the relative motion of the aircraft, the droplet velocity can be calculated by using the local approximation method and the droplet velocity model in a three-dimensional coordinate system, as follows ${ }^{[17]}$ :

$$
\begin{gathered}
V_{d x}=V_{x 0} e^{-\lambda S_{x}} \\
V_{d y}=V_{d y 0} e^{-\lambda S_{y}}-\left(1-e^{-\lambda S_{y}}\right) g \lambda \\
V_{d z}=V_{f}-\left(V_{f 0}-V_{d z 0}\right) e^{-\lambda S_{z}}
\end{gathered}
$$

and $\lambda$ is defined by

$$
\lambda=\frac{3 C_{d} \rho}{8 \rho_{d} d}
$$

where, $V_{d x}, V_{d y}$, and $V_{d z}$ represent the instantaneous velocity of droplets in $x$-, $y$-, and $z$-directions, respectively, $\mathrm{m} / \mathrm{s} ; V_{d x 0}, V_{d y 0}$, and $V_{d z 0}$ represent the initial velocity of droplets in $x$ -,$y$-, and $z$-directions, respectively, $\mathrm{m} / \mathrm{s} ; V_{f 0}$ is the velocity of airflow near the nozzle, $\mathrm{m} / \mathrm{s} ; \lambda$ represents the momentum relaxation coefficient; $S_{x}$, $S_{y}$, and $S_{z}$ represent the movement distance of droplets in $x$-, $y$-, and $z$-directions, respectively, $\mathrm{m}$.

As illustrated in the above models, the instantaneous velocity of droplets in $x$-, $y$-, and $z$-directions decreases with the increase of the movement time and displacement distance of droplets. When the velocity of a droplet is almost equal to 0 , the movement of droplets will end. At this time, the movement displacement of droplets in the three-dimensional direction can be calculated, and the drift distance of droplets can be known.

\subsection{Factors that influence spray drift}

Understanding the factors influencing droplet drift can not only reduce droplet drift and improve the utilization rate of pesticides by selecting the best operating environment and flight parameters, but also help us better understand the influencing mechanism of factors and the law of droplet drift and deposition, which will contribute to the improvement of aerial spray system and the development of DRTs. In the process of atomization and deposition of liquid medicine, many factors are affecting the droplet drift, including the droplet size parameters, the type of aircraft and nozzle, the physical and chemical properties of the liquid, and the meteorological conditions and operating parameters.

\subsubsection{Droplet size}

According to the American Society of Agricultural Engineers (ASAE) S-572 standard (S-572 Spray Tip Classification by Droplet Size), droplets can be classified into six categories according to particle size: Very-Fine droplet (less than $100 \mu \mathrm{m}$ ), Fine droplet (100-175 $\mu \mathrm{m})$, Medium droplet $(175-250 \mu \mathrm{m})$, Coarse droplet (250-375 $\mu \mathrm{m})$, Very-coarse droplet (375-450 $\mu \mathrm{m})$ and Extra-coarse droplet (greater than $450 \mu \mathrm{m}$ ). Studies have shown that droplet size is the most important factor causing spray drift among the affecting factors ${ }^{[18]}$. The smaller the droplet, the longer it floats in the air and the more effective it is in drifting with the wind ${ }^{[19]}$. The falling speed of a fine droplet is decreasing substantially under the air resistance, which leads to the droplet not having enough downward momentum to reach the target. Conversely, fine droplets are more susceptible to temperature and relative humidity in the air, and the droplet size is further reduced after evaporation, which can drift far away with the wind ${ }^{[20]}$. The suspension time required for droplets of different particle sizes to drop by $3 \mathrm{~m}$ as Table $1^{[21]}$. 
Table 1 Effects of droplet sizes on drift potential

Droplet diameter $\mu \mathrm{m} \quad$ Suspension time required for droplets to drop by $3 \mathrm{~m} / \mathrm{s}$

$\begin{array}{cc}5 & 3960 \\ 20 & 252 \\ 100 & 10 \\ 240 & 6 \\ 400 & 2 \\ 1000 & 1\end{array}$

Using a computer model, Hobson et al. ${ }^{[22]}$ determined the proportion of drift that would increase sharply when droplets were less than $100 \mu \mathrm{m}$. Due to evaporation, the diameter of a $100 \mu \mathrm{m}$ droplet moving $75 \mathrm{~cm}$ at $25^{\circ} \mathrm{C}$ and $30 \%$ relative humidity was reduced by $50 \%$. Under the same climatic conditions, droplets less than $100 \mu \mathrm{m}$ were volatilized into smoke and suspended in the atmosphere before reaching the target, eventually landing in a non-target area. Droplets larger than $200 \mu \mathrm{m}$ did not evaporate easily due to the smaller relative surface area, resulting in a faster falling speed and better drift resistance than smaller droplets ${ }^{[23]}$. Bird et al. ${ }^{[18]}$ summarized the completed aerial spraying test and found that the drift rate of droplets with a volume median diameter (VMD) of less than $200 \mu \mathrm{m}$ was 5-10 times greater than droplets with a VMD of more than $500 \mu \mathrm{m}$. This conclusion has also been demonstrated by Yates et al. ${ }^{[24]}$, who showed that the horizontal drift of droplets with a VMD of $290 \mu \mathrm{m}$ was greater than twice that of droplets with a VMD of $420 \mu \mathrm{m}$. Yates et al. ${ }^{[25]}$ also found that the drift of droplets with a VMD of $175 \mu \mathrm{m}$ was approximately 5.5 times greater than droplets with a VMD of $450 \mu \mathrm{m}$. Hewitt et al. ${ }^{[26]}$ studied the effects of five droplets ranging from very-fine to very-coarse on deposition and drift using a Cessna AgHusky manned aircraft (US). The authors found that there were significant differences in drift across the five different particle sizes and the amount of drift decreased significantly with the greater particle size. Using a wind tunnel, Wang et al. ${ }^{[11]}$ tested the droplets of different sizes sprayed by agricultural unmanned aerial vehicles (UAV) and showed that small droplets with a VMD of $101.74 \mu \mathrm{m}$ are more likely to drift at the same wind speed, drift volume and drift distance of them were significantly larger than droplets with a VMD of $164.00 \mu \mathrm{m}$ and $228.16 \mu \mathrm{m}$. Also using a wind tunnel, Ru et al. ${ }^{[17]}$ analyzed the factors affecting droplet drift under wind tunnel conditions based on the established droplet drift model, the results showed that in the direction of airflow, the maximum drift distance of droplets with a VMD of $60 \mu \mathrm{m}$ was $30.25 \mathrm{~m}$ and the maximum drift distance of the droplets with a VMD of $150 \mu \mathrm{m}$ was $10.76 \mathrm{~m}$. What's more, compared with small droplets, the drift of large droplets was reduced by nearly $1 / 3$. All the above studies have validated the significant effect of droplet size on droplet drift from theoretical and experimental perspectives. 2.2.2 Meteorological parameters

Meteorological parameters are important factors that cannot be ignored in the study of droplet deposition and drift. In the process of droplet deposition from the nozzle to the ground, droplets are susceptible to temperature and relative humidity, and evaporation causes droplets to become smaller and more likely to drift to a greater extent in the natural wind $^{[20]}$. Therefore, the main meteorological parameters affecting droplet deposition and drift are natural wind, temperature and humidity, and atmospheric stability.

1) Wind

Wind speed and wind direction have great influences on the horizontal movement of droplets in the air, and determine the horizontal movement speed and direction of droplets. Studies show that the relationship between wind speed and droplet drift is nearly linear ${ }^{[27]}$. Grover et al. ${ }^{[28]}$ conducted research on aerial spraying using a manned aircraft and found that when the wind speed was $10 \mathrm{~km} / \mathrm{h}$, the drift ratio of droplets was about $22 \%$. When the wind speed was $25 \mathrm{~km} / \mathrm{h}$, the drift ratio of droplets has increased by more than $10 \%$. Thistle et al. ${ }^{[29]}$ summarized previous studies demonstrating that the increase in wind speed can lead to a larger proportion of droplet drift. As shown in Table 2, a greater wind speed would cause droplets to drift farther. Wang et al. ${ }^{[30]}$ used wind tunnel tests to study the effects of different wind speeds of $1,2,3$, and $4 \mathrm{~m} / \mathrm{s}$ on the droplet deposition and drift of aerial sprays by agricultural UAV. It was found that wind speeds have an extremely significant effect on droplet deposition and drift and that when the wind speed was greater than $4 \mathrm{~m} / \mathrm{s}$, the drift increased sharply. Wang et al. ${ }^{[31]}$ measured the drift characteristics of a fuel-powered single-rotor UAV for plant protection and found that the cumulative drift rate of droplets ranged from $14.3 \%-75.8 \%$ when the crosswind speed ranged from $0.76-5.50 \mathrm{~m} / \mathrm{s}$, indicating that the drift rate of droplets strongly and positively correlated with wind speed. Zhang et al. ${ }^{[32]}$ studied the drift of droplets in non-target areas under the crosswind speed of 1 , 2 , and $3 \mathrm{~m} / \mathrm{s}$, and showed that the maximum drift distance and deposition location of the liquid varied significantly with the crosswind speed.

Table 2 Drift distance of droplets with different size in a $3.048 \mathrm{~m}$ fall in different wind speed environment

\begin{tabular}{ccc}
\hline \multirow{2}{*}{$\begin{array}{c}\text { Wind speed } \\
/ \mathrm{m} \cdot \mathrm{s}^{-1}\end{array}$} & \multicolumn{2}{c}{ Drift distance $/ \mathrm{m}$} \\
\cline { 2 - 3 } & $100 \mu \mathrm{m}$ (Very-fine droplet) & $400 \mu \mathrm{m}$ (Very-coarse droplet) \\
\hline 0.45 & 4.7 & 0.9 \\
2.23 & 23.5 & 4.6 \\
\hline
\end{tabular}

2) Temperature and humidity

Temperature and humidity with regard to spray drift influence droplet size. Droplet evaporation is accelerated in low humidity and high-temperature environments during the deposition process, and high-temperature environments may also increase the volatilization of pesticide products. As a result, droplets become smaller and easier to suspend in the air or drift. Luo et al. ${ }^{[33]}$ observed that at a constant temperature of $25^{\circ} \mathrm{C}$, a $1070 \mu \mathrm{m}$ droplet took $300 \mathrm{~s}$ to completely evaporate at $20 \% \mathrm{RH}$, and $540 \mathrm{~s}$ to completely evaporate at $60 \% \mathrm{RH}$. Luo et al. ${ }^{[34]}$ also demonstrated that a $910 \mu \mathrm{m}$ droplet at $60 \% \mathrm{RH}$ took $780 \mathrm{~s}$ to evaporate at $10^{\circ} \mathrm{C}$, but only $420 \mathrm{~s}$ to evaporate completely at $25^{\circ} \mathrm{C}$. Understanding the principle and process of droplet size reduction is an important prerequisite for modeling droplet deposition and drift. For that reason, a series of mathematical models have been developed to predict the evaporation rate of droplets during the falling process. The earliest modeling work was conducted by Ranz et al. ${ }^{[35]}$, which was later optimized by Williamson et al. ${ }^{[36]}$, whose model is utilized by Agricultural Dispersal (AGDISP) model for aerial spraying conducted commonly today. An example of the application of the model is discussed by Picot et al. ${ }^{[37]}$, who showed that an $85 \mu \mathrm{m}$ droplet falling through an environment with a temperature of $10^{\circ} \mathrm{C}$ and $\mathrm{RH}$ of $60 \%$ would reduce to one-half of its original size in $107 \mathrm{~s}$.

3) Atmospheric stability

Atmospheric stability refers to the degree to which an air mass rises or falls due to the buoyancy generated by the difference in intensity, temperature, and flow velocity from the surrounding air. Under stable atmospheric conditions with lower atmospheric mixing characteristics, droplets will not deposit into the lower cool 
air, or disperse to the upper layer, and tend to remain suspended in the stable airflow. Under these conditions, the droplet mass in stable atmospheric slowly moves to a sensitive region in any direction. However, once the steady-state is destroyed, a large number of droplets may move and drift $^{[38]}$. Many studies show that a relatively stable atmospheric environment will increase the drift potential of liquid droplets. Using a spray test, Yates et al. ${ }^{[39]}$ observed that as the atmospheric stability decreased (very stable to unstable), so did the droplets moving in a downwind direction. In addition, the larger the drift distances of droplets are, the greater the influence of atmospheric stability on droplet deposition and drift. Miller et al. ${ }^{[40]}$, in reviewing the work of Yates et al. ${ }^{[24]}$, concluded that atmospheric stability plays a dominant role in the deposition and drift of droplets in the far field. Using an airblast sprayer for orchard applications, Miller et al. ${ }^{[41]}$ reported that there was an increase in droplet deposition in stable conditions compared to unstable conditions. Fritz et al. ${ }^{[42]}$ found that atmospheric stability has a greater impact on smaller droplets, and the suspending time of smaller droplets than larger droplets, and that the suspension time of smaller droplets in the air is longer with greater atmospheric stability, which will affect the deposition and drift of droplets. Hoffmann and Salyani ${ }^{[43]}$ reported higher downwind ground depositions at nighttime versus daytime application, and found that the most stable atmospheric conditions occurred at night, in agreement with earlier findings.

\subsubsection{Nozzles}

The nozzle is an important component of the spraying system for plant protection and is also the key factor in controlling droplet size, which directly affects the spray quality and deposition characteristics. During the spraying process, the nozzle determines the droplet size spectrum, including droplet size, the span of size and other characteristics, thus affecting the droplets deposition and drift in the crop canopy ${ }^{[44,45]}$. Nuyttens et al. ${ }^{[46]}$ tested different types of Hardi flat spray nozzles together with the five reference nozzles by using PDPA. In total, 32 nozzle-pressure combinations were evaluated. The authors found that the nozzle type and pressure had a significant effect on the droplet size and velocity. Presently, the most common types of nozzles on the market are the hydraulic nozzle and the centrifugal nozzle. Compared with the hydraulic nozzle, the centrifugal nozzle produces a narrower particle size spectrum, indicating a more uniform droplet size. However, Bouse et al. ${ }^{[47]}$ proposed that the hydraulic nozzle could also produce a droplet size spectra with an ideal target VMD, and with a small percentage of spray volume contained in droplets less than $100 \mu \mathrm{m}$ in diameter. Conversely, studies have shown that there is a correlation between droplet size and spray pressure. Larger droplets tend to be produced by lower pressure units, which can reduce spray drift.

Another factor affecting the drift of droplets is the spray angle. Increasing the nozzle angle could reduce the drop size. Miller et al. ${ }^{[48]}$ measured the droplet size and velocity distributions for the reference nozzles with different angles, and showed that the mean liquid velocity for the nozzle with a $60^{\circ}$ angle was less than that nozzles at a $55^{\circ}$ angle. Measurements also were made with three nozzle spray fans, angled at $65^{\circ}, 80^{\circ}$, and $110^{\circ}$. Results of this work showed that the use of nozzles with fan angles less than $110^{\circ}$ can reduce the risk of drift.

\subsubsection{Operating parameters}

Numerous tests and studies have shown that the operation parameters of aerial spraying have a significant impact on droplet and drift, and that the drift of droplets decreases with a reduction in flight height and speed. When the aircraft has a higher flight altitude and a faster flight speed, the downward wind field below the aircraft is weakened and the influence of crosswind is stronger, encouraging drift of droplets ${ }^{[49,50]}$. Moreover, as airspeed increases, so does air shear that shatters the large droplets, increasing the percentage of fine or very-fine droplets and the likelihood of drift ${ }^{[51]}$. According to the results of Jong et al. ${ }^{[52]}$ and Nuyttens et al. ${ }^{[53]}$, reducing the spraying height can $20 \mathrm{~cm}$ would significantly decrease the amount of droplet drift by $54.0 \%$ and $40.1 \%$, respectively. In addition, the effect of the operating speed on droplet drift was also tested, and it was found that droplet drift significantly increased at higher operating speeds ${ }^{[54,55]}$. Tang et al. ${ }^{[56]}$ simulated the effect of flight speed on the droplet size from spray operation of a manned aircraft using a wind tunnel test, and showed that the VMD of the droplets decreased rapidly (by about $70 \%$ ) when the flight speed increased from 120 to $305 \mathrm{~km} / \mathrm{h}$. They also showed that the amount of droplet drift increased significantly when the flight speed exceeded $305 \mathrm{~km} / \mathrm{h}$. Huang et al. ${ }^{[57]}$ compared the droplet deposition and drift results of the manned aircraft at three different flight altitudes of 3.7, 4.9, and $6.1 \mathrm{~m}$, and found that when the flight height was $6.1 \mathrm{~m}$, the drift of droplet in the downwind direction was at its maximum. Using a spray test with different flight parameters of plant protection UAV in rice, Chen et al. ${ }^{[58]}$ found that flight parameters had a significant effect on droplet deposition and drift, with an increase in flight height leading to an increase in the amount of drift and drift distance in the drift area. Xue et al. ${ }^{[50]}$ also reached similar conclusions in spray tests of the oil-powered UAV under different flight parameters.

\subsubsection{Physicochemical Properties of liquid medicine}

The physicochemical properties of liquid pesticides are important factors affecting the deposition and drift of droplets. The physicochemical parameters affecting the deposit mechanism of droplets primarily include viscosity, surface tension, and inhomogeneity of liquid medicine. Presently, the physicochemical properties of liquid pesticides are generally changed by adding chemical adjuvants. Developed countries such as Europe and the United States have carried out a number of studies on spray drift with the formulation of pesticide adjuvants. To reduce spray drift, polymer-type adjuvants are widely used in the United States and Australia. Studies have shown that adding adjuvants to a solution changes the droplet size by modifying the dynamic surface tension and other physical properties of the solution. Conversely, adjuvants can effectively reduce the evaporation of droplets during the deposition process to inhibit the droplet size reduction. Wind tunnel tests have shown that spraying a solution of surfactant and emulsion causes air entrainment to drift, and the emulsion can increase the velocity and the collision probability of droplets, narrowing the width of the droplet size spectrum ${ }^{[1,60]}$. Miller et al. ${ }^{[38]}$ studied the influence mechanism of adjuvants on droplet size, droplet velocity and droplet size spectrum, and compared the drift of the droplets after adding different adjuvants. The results showed that different types of adjuvants affected droplet deposition and drift differently. Lan et al. ${ }^{[61]}$ obtained similar results when looking at four different types of adjuvants and water applied using an agricultural manned aircraft. Three types of aerial spray adjuvants were compared and evaluated, including macromolecule sugar and organosilicon, and it was found that adding adjuvants significantly prolonged the evaporation time of droplets, slowing the speed of droplet size reduction and reducing the drift of small droplets. 


\section{Research status of DRTs in aerial spraying}

\subsection{Aerial electrostatic spraying technology}

Aerial electrostatic spraying technology is an innovative application of traditional ground electrostatic spraying technology in combination with a $\mathrm{UAV}^{[62,63]}$. In the process of aerial spraying, the electrostatic field between the nozzle and the target crop is established by high-voltage static electricity, which makes pesticide liquid charge with the same polarity as nozzle after atomizing. According to the principle of electrostatic induction, the ground plant canopy causes an opposite charge of droplets. As the same-sex charges inter-repel and the opposite-sex charges inter-attract, the charged droplets will make directional movements to the ground and eventually attach to various parts of the target crop plants with the effect of electrostatic field forces and other external forces ${ }^{[64]}$. Electrostatic spraying technology not only makes droplets attach not only on the front of the crop leaves but also in the middle and lower parts of the plants and the back of leaves, which increase the droplets' deposition rate and spray coverage while reducing droplet drift and improve the ecological environment around the application area.

The application of electrostatic spraying technology in agricultural aviation began in the $1960 \mathrm{~s}$. Calton et al. ${ }^{[65-67]}$ conducted a study on aerial electrostatic spraying technology and developed an electric rotary nozzle designed to reduce spray drift. The results showed that electrostatic spraying technology can somewhat decrease droplet drift to a certain degree by accelerating the deposition process of charged droplets and increasing the penetration of droplets in the crop canopy. To better test the effect of droplet charging and fuselage discharging, Calton et al. ${ }^{\text {[68] }}$ developed a droplet detector to understand the deposition process of charged droplets and the deposition effect on target crops, and also sought to validate the potential application of aerial electrostatic spraying technology by reducing droplet drift in laboratory and field experiments. Since, with the development of electronic technology and computer technology, aerial electrostatic spraying system has been greatly improved and field tested ${ }^{[69,70]}$. $\mathrm{Ru}$ et al. ${ }^{[71]}$ designed an aerial electrostatic spraying system for Y5B aircraft and conducted experimental studies examining the effective spraying width, droplet deposition characteristics, and drift. Compared with conventional spraying methods, electrostatic spraying can increase the deposition density of droplets by $14 \mathrm{pcs} / \mathrm{cm}^{2}$, and droplet drift is significantly reduced. Based on their research, $\mathrm{Ru}$ et $\mathrm{al}^{\left[{ }^{[72]}\right.}$ designed an electrostatic spraying system to be used for UAVs for the first time. A preliminary experiment showed that the droplet deposition and drift characteristics have been greatly improved under electrostatic action, which proved the feasibility of electrostatic spraying technology applied to UAVs.

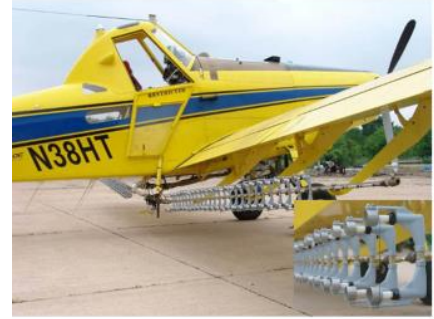

a. Electrostatic spray system for manned aircraft ${ }^{[62]}$

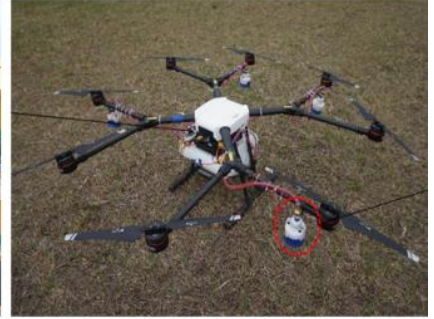

b. Electrostatic spray system for UAV
Note: Electrostatic nozzle was marked in the red circle in Figure 1b. Figure 1 Aerial electrostatic spray system

\subsection{Aerial spray adjuvant}

Spray adjuvants are substances that are used to improve the physicochemical properties of solutions during spray applications, and the physicochemical properties of solutions are important factors affecting spray quality. Adding adjuvants to the spray solution can change the droplet size by changing the dynamic surface tension and other physical properties of the solution. It can effectively reduce the evaporation of the droplet in the deposition process to inhibit the droplet size reduction ${ }^{[73]}$. Tests show that reasonable use of spray adjuvant can reduce droplet drift in the downwind direction by about $50 \%{ }^{[20]}$. With the wide application of agricultural aerial spraying, the spray adjuvant is one of the effective ways to achieve high-efficiency pesticide use and solve the problem of droplet drift. At present, the United States, Australia, and other developed countries generally need to add spray adjuvants in pesticide spraying operations, which can increase droplet deposition and reduce droplet drift, and improve the use effect of pesticides. Studies have shown that pesticide adjuvants and formulations affect the atomization performance of the nozzle. The addition of adjuvants will significantly change the droplet size and liquid film thickness, which increase the deposition rate of droplets and reduce the drift rate of droplets ${ }^{[74]}$. Dexter et al. ${ }^{[75]}$ found that with the increase of the concentration of adjuvants, the droplet size increased and the proportion of fine or very-fine droplets decreased significantly. Wang et al. ${ }^{[76]}$ compared and analyzed the influence of different concentrations of spray adjuvants on the drift potential index of nozzles. The results showed that compared with water, the three adjuvants could reduce the drift potential index by $98.7 \%, 58.2 \%$ and $80.1 \%$, respectively. The experimental results also verified that the drift of droplets is closely related to the volume percentage of fine droplets, which provided a theoretical basis for further research on new spray adjuvants and reducing droplets drift.

\subsection{Aerial air-assisted spraying technology}

Air-assisted spraying technologies refer to the use of airflow generated by an assistant airflow device to transport droplets to the target crop during spraying. The airflow carrying fine droplets reaches both the front and back sides of the leaves, increasing the coverage density and uniformity of pesticides on the target crop, significantly reducing the drift of droplets and improving the utilization rate of pesticides ${ }^{[77]}$. Nordbo et al. ${ }^{[78]}$ showed that airflow can improve the size spectrum of small droplets and reduce the drift of droplets, as well as improve the stability of low-volume spraying. He et al. ${ }^{[79]}$ studied the effect of airflow of airborne sprayers on droplets deposition, and showed that the permeability and deposition of droplets in the canopy are positively correlated with wind speed. The higher the wind speed, the better the penetration of droplets, and the more droplets deposited on the target crops, which can effectively control the drift of pesticide droplets. The JT30 type plant protection UAV (Xinjiang Sky Aviation Technology Co., Ltd, China) is the first application of air-assisted spray technology for UAVs. As shown in Figure 2, the droplets sprayed from the nozzles become more uniform after the liquid is atomized by the airflow generated by the high-pressure centrifugal fan. Under the assisted influence of airflow, it not only increases the deposition in the middle and lower layers of plants and the penetration of the droplets but also can reduce the drift of droplets and use pesticide effectively. The ductal pesticide spraying device is based on a multi-rotor agricultural UAV (the National Center for International Collaboration Research on Precision Agricultural Aviation Pesticides Spraying Technology, 
China) and has a similar spraying effect, as shown in Figure 2. Droplets are transported to the target crops by directional airflow generated by ducted fans under UAV, and smaller droplets are easier to deposit under the assisted influence of airflow, reducing droplets drift and environmental pollution.

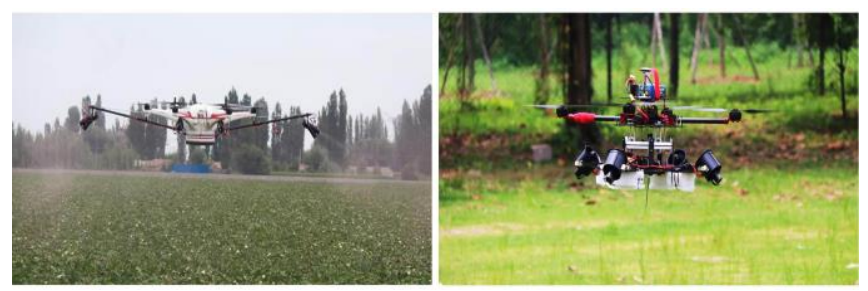

Figure 2 Aerial air-assisted spray for plant protection UAV

\subsection{Drift reducing nozzle}

The drift reducing nozzle is an important part of agricultural aircraft to achieve a good spray effect during the application process, and is an important factor to ensure the effect of spraying. It directly affects the quality of spray, and determines the reliability and economy of the whole plant protection spraying system. During the spraying process, the nozzle determines the distribution of the droplet size spectrum, including droplet size, span of droplet size and other characteristics, thus affecting the droplets deposition and drift in crop canopy. At present, the drift reducing nozzle mixes air and water in the nozzle based on jet technology to form two-phase flow and then atomize into droplets. When the liquid passes through the contraction section of the inner core of nozzle, the flow velocity of liquid is increasing rapidly. After the liquid is ejected from the compression section, the high-speed flowing liquid takes away the surrounding air and forms a vacuum area near the outlet of the compression section. The air being sucked in the compression section would be mixed with the liquid and exchanged energy, and the two-phase flow liquid entered the diffusion section together. Then the large droplets with bubbles were ejected through the nozzle. It can reduce the proportion of the droplets that are easy to drift and achieve the goal of reducing the pesticide droplets drift ${ }^{[31]}$.

In recent years, the United States Lurmark Inc., the Germany Lechler Inc., and other companies have designed many types of drift reducing nozzles. Among them, ID/IDK/IDKT drift reducing jet nozzles (Figure 3) have more uniform droplet coverage and low droplet drift, which the drift reducing effect can reach more than $95 \%$ at a wind speed of $12-28 \mathrm{~km} / \mathrm{h}$ and still more than $70 \%$ at a wind speed of $35 \mathrm{~km} / \mathrm{h}$. The atomization characteristics (droplet size, droplet velocity, etc.) of the conventional hydraulic nozzle and air-suction drift reducing nozzle were compared by Dorr et al. ${ }^{[4]}$, it was found that the droplets produced by air-suction drift reducing nozzle had larger droplet size and slower deposition velocity, which were not easy to drift. Song et al. ${ }^{[80]}$ studied the droplet drift mechanism of fan-shaped nozzle, the test showed that the droplets which are easily drifting in the spray process were mainly concentrated in the center of the spray fan from the nozzle of 300-500 mm, and the drift area were the end and sides of the spray fan. It indicated that the influence of these factors on spray drift of nozzles should be considered in the process of designing the drift reducing nozzles. Tang et al. ${ }^{[55]}$ tested the LU-120-03 standard fan nozzle and the IDK-120-03 air-induced nozzle in the wind tunnel. The results showed that the air-induced nozzle had a larger droplet size and better uniformity of droplets within a wide range of wind speed, and was more suitable for use as an aerial spraying nozzle.

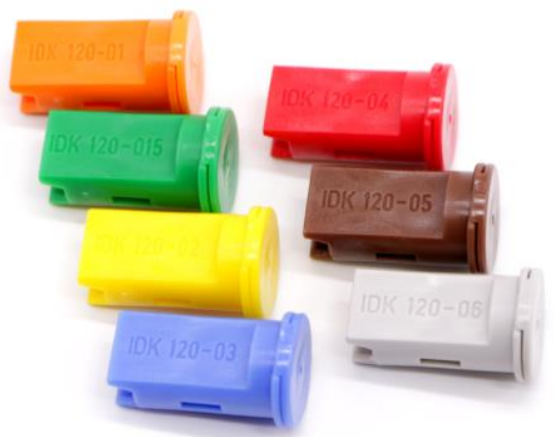

Figure 3 IDK series drift reducing jet nozzles

\subsection{Aerial variable-rate spraying technology}

Aerial variable-rate spraying technology is one of the means to achieve precise spraying, which is able to increase the precision of plant protection spray applications. With this technology, crops are able to be sprayed for different purposes by synthesizing information about target crops, such as field pest and disease area, crop row spacing and plant density and other application parameters ${ }^{[81]}$. Compared to traditional high-capacity spray technology, variable-rate spraying technology can reduce the problem of pesticide overuse, reduce droplet drift, and improve the efficiency of pesticides.

In recent years, the availability and use of variable-rate application systems, such as the AG-FLOW system developed by Canada AG-NAV Inc., has increased ${ }^{[82]}$. The system can display the spraying area and the flight route in real-time, and control the spraying flow according to flight parameters. In 2016, the precision agricultural aviation team of South China Agricultural University tested the system on the AS350B3e helicopter, verified the system's effectiveness, as shown in Figure $4^{[83]}$. The Wingman GX system was developed by Adapco Inc. provides basic flight guidance, flight records and spraying flow-rate control and can potentially be used to reduce the amount of pesticide drift beyond the intended area through accurate analysis of meteorological information and maximizing the quality of spray. The Satloc M3 system developed by Hemisphere Inc., which is primarily used with the AirTrac software and the AerialAce flow controller, was tested by Thomson et al. ${ }^{[84]}$ on an Air Tractor 402B aircraft and analyzed for flow control accuracy and swath deposition positioning. And the Satloc G4 system was tested by the precision agricultural aviation team of South China Agricultural University in 2016 in Jiamusi, Heilongjiang Province, China, as shown in Figure 4. In addition, in order to avoid the problems of the low utilization rate of pesticides and environmental pollution caused by plant protection UAV spraying, Wang et al. ${ }^{[85]}$ developed a precision spraying control system for UAV based on image recognition, which used the algorithm to classify and identify the crop area and non-crop area in the aerial image of the field, and controlled the nozzle based on recognized results to

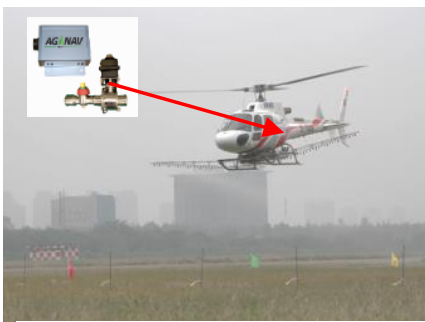

a. Variable-rate spray system for manned aircraft

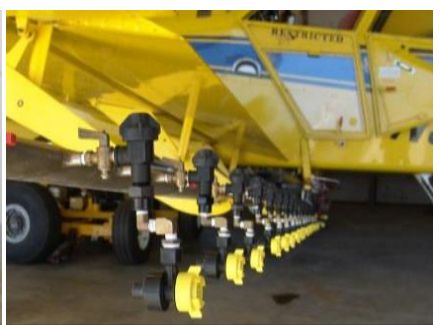

b. Aerial variable-rate nozzle
Figure 4 Aerial variable-rate spray 
achieve precise spray. The application of aerial variable-rate spraying technology has provided a technical reference for research and provide reference guides for the development of the DRTs in the future.

\section{Suggestions and countermeasures for drift reduction in aerial spraying}

\subsection{Strengthening the research on DRTs for plant protection} UAV

1) Improvement of electrostatic spraying and variable-rate spraying technologies

Electrostatic spraying and variable-rate spraying as one of the means to achieve precise spraying of pesticides, which can effectively reduce droplet drift and improve the utilization rate of pesticides. It is considered to be an important development direction in agricultural aviation spraying in the future. Presently, electrostatic spraying technology and variable-rate spraying technology have been maturely applied to equipment that is used on the ground plant protection machinery and the agricultural manned aircraft, and are only in preliminary stages for plant protection UAV. As one of the important parts of agricultural aviation equipment, plant protection UAV has great operational advantages compared with other machinery, but due to the small capacity of plant protection UAV and the separation from the ground, the application of electrostatic spraying technology and variable-rate spraying technology in plant protection UAV is currently limited. There are still many problems in the application of its technologies and devices in plant protection UAVs to be solved. Therefore, in order to take advantages of plant protection UAVs with low or ultra-low capacity spraying and to improve pesticide waste and environmental pollution, plant protection UAVs should be optimized with electrostatic spraying technology and variable-rate spraying technology in the future. Their application for precision agriculture also needs to be promoted.

2) Research and development of aviation-specific adjuvants

Adjuvants with good drift reduction and evaporation prevention are important conditions for ensuring the effect of agricultural aviation application. Developed countries in Europe and the United States have carried out a large number of studies on the effects of the formulation of adjuvants on droplets drift during spraying, but the high-efficiency adjuvants for UAV application of pesticides, especially for low or ultra-low capacity spraying, are still not well developed. Therefore, the effects of different emulsifiers, dispersants, solvents, and other components on the physicochemical parameters for aerial application need to be studied, especially for rice, wheat, and maize, and select the adjuvants for aerial spraying that can reduce droplets drift, prevent droplet evaporation and promote the adhesion and spreading of pesticide droplets.

3) Design of key components for spraying

The performance of key components for spraying directly affects the atomization quality of aerial spraying, and key components with good performance can minimize the drift of the pesticide droplets. At present, the key components of the spraying system for agricultural manned aircraft have been tested extensively and applied maturely. However, plant protection UAVs are a new type of plant protection device, and spraying components for these have few species, simple structure and single function. Many of the spraying components of plant protection UAVs have been transplanted from the spraying system of the ground plant protection machinery and the manned aircraft. There is no specific operating system and standardized pattern for plant protection UAVs, which often causes a large number of pesticide droplets to drift during the spraying process. Therefore, other advanced technology abroad should be fully used for reference and introduction, and combined with the DRTs to strengthen the research and development of special spraying components for plant protection UAVs.

\subsection{Adoption of reasonable drift reducing method}

To minimize the droplets drift of aerial spray, in addition to using some drift reducing techniques and means, some reasonable drift reducing methods can be adopted:

1) Reasonable selection of spraying operation parameters

The spraying operation parameters (flight height and speed) of the aircraft determine the deposition time and distance of droplets in the air, affecting the drift distance of droplets. Studies have shown that the optimal operating parameters are different for different types of crops. Therefore, within the range of operating parameters allowed by the aircraft, the reasonable operating parameters of aerial spraying should be selected as far as possible to reduce the settling time of droplets from the nozzle to the target and the probability of off-target of droplets to reduce the droplets drift.

2) Suitable selection of spraying time

Meteorological factors are important factors that cannot be ignored in dictating droplet deposition and drift. To effectively reduce the drift of pesticide droplets, the applicator must keep abreast of the weather conditions when spraying pesticides, such as wind speed and wind direction, temperature, humidity, and other climatic conditions. Pesticide droplets are easily evaporated and drift under high temperature and low humidity. Therefore, it is recommended to measure the environmental parameters such as wind speed, wind direction, and temperature and humidity, before the aerial spraying operation to select the most suitable time for the spraying operation. Especially in summer, as the outbreak period of diseases, insects and weeds in a year and the critical period of spraying operation, the best time for spraying operation should be selected before 11:00 a.m. and after 4:00 p.m. to reduce the waste and drift of the pesticide droplets caused by evaporation and drift.

3) Security buffer zone settings

Pesticide drift is inevitable for spraying operations, especially for aerial spraying with higher flight altitude and wind field generated by UAV's rotor. Therefore, when the target crop is adjacent to a water source, developed area, or sensitive animals and plants, a buffer zone should be included between the target crop and the sensitive area before the aerial spraying operation to reduce and prevent the phytotoxicity of pesticides caused by the droplets drift. Furthermore, the width of the security buffer zone should be set and adjusted according to the pesticide formulations, the species of the sensitive area, the droplets size generated by the spray system, and the environmental parameters during spraying application.

\section{Conclusions}

With the continuing deterioration of the ecological environment and the improvement of people's requirements for modern life quality, the problems of pesticide pollution and residues will be paid more and more attention, and the use of pesticides will inevitably develop towards higher utilization rate of pesticides and less environmental pollution. Based on an analysis of the spray drift mechanism and a summary of previous studies, it 
was found that factors such as droplet size, meteorological parameters, nozzles, operating parameters and physicochemical properties of liquid medicine all affect the droplet deposition and drift of aerial spraying. Thus, an effective combination of low- or ultra-low-volume spraying operation mode of aerial spraying and DRTs is an important means to achieve this goal.

At present, research has been conducted on aerial spray technologies, including aerial electrostatic spraying technologies, aerial air-assisted spraying technology, aerial spraying adjuvant, drift reducing nozzles, and aerial variable-rate spraying technology, which has made some progress. However, there are still some problems that have not been solved, especially when it comes to deploying plant protection UAVs. To reduce droplet drift of agricultural aviation spraying, one is necessary to strengthen the research and improvement on DRTs for plant protection UAVs, and the other is to adopt reasonable DRTs methods.

Therefore, the research on the combination of agricultural aviation spraying technology and DRTs should continue to be strengthened in the future, so as to give full play to the advantages of precision agricultural aviation technology in reducing pesticide use and residues, improve pesticide use efficiency, and minimize environmental pollution caused by pesticide drift.

\section{Acknowledgements}

The authors acknowledge LetPub (http://www.letpub.com) for its linguistic assistance during the preparation of this manuscript. This work was financially supported by the National Natural Science Foundation of China (Grant No.31901411); the Science and Technology Planning Project of Guangdong Province (Grant No. 2019B020208007); the Young Innovative Talents Project of Regular Institutions of Higher Education of Guangdong Province (Grant No. 2018KQNCX020); Science and Technology Planning Project of Guangzhou (202103000090); the Key R\&D projects in Hainan Province (ZDYF2020195).

\section{[References]}

[1] Hilz E, Vermeer A W P. Spray drift review: The extent to which a formulation can contribute to spray drift reduction. Crop Protection, 2013 44(1): 75-83.

[2] Lan Y B, Chen S D, Fritz B K. Current status and future trends of precision agricultural aviation technologies. Int J Agric \& Biol Eng, 2017; 10(3): $1-17$.

[3] Glass C R, Walters K F, Gaskell P H, Lee Y C, Thompson H M, Emerson D $\mathrm{R}$, et al. Recent advances in computational fluid dynamics relevant to the modelling of pesticide flow on leaf surfaces. Pest Management Science, 2010; 66(1): 2-9.

[4] Yuan H Z, Yang D B, Yan X J, Zhang L N. Pesticide efficiency and the way to optimize the spray application. Plant Protection, 2011; 37(5): 14-20. (in Chinese)

[5] Chen S D, Lan Y B, Li J Y, Xu X J, Wang Z G, Peng B. Evaluation and test of the effective spraying width of aerial spraying on plant protection UAV. Transactions of the CSAE, 2017; 33(7): 82-90. (in Chinese)

[6] Shamshiri R R, Hameed I A, Balasundram S K, Ahmad D, Weltzien C, Yamin M. Fundamental research on unmanned aerial vehicles to support precision agriculture in oil palm plantations. INTECH: Agricultural Robots: Fundamentals and Applications, 2018; pp.91-116.

[7] Xue X Y, Lan Y B, Sun Z, Chang C, Hoffmann W C. Develop an unmanned aerial vehicle based automatic aerial spraying system. Computers and Electronics in Agriculture, 2016; 128: 58-66.

[8] Zhou Z Y, Zang Y, Luo X W, Lan Y B, Xue X Y. Technology innovation development strategy on agricultural aviation industry for plant protection in China. Transactions of the CSAE, 2013; 29(24): 1-10. (in Chinese)

[9] Lan Y B, Chen S D. Current status and trends of plant protection UAV and its spraying technology in China. Int J Precis Agric Aviat, 2018;
1(1): $1-9$.

[10] Liao J, Zang Y, Zhou Z Y, Luo X W. Quality evaluation method and optimization of operating parameters in crop aerial spraying technology. Transactions of the CSAE, 2015; 31(S2): 38-46. (in Chinese)

[11] Wang X N, He X K, Wang C L, Wang Z C, Li L L, Wang S L, et al Spray drift characteristics of fuel powered single-rotor UAV for plant protection. Transactions of the CSAE, 2017; 33(1): 117-123. (in Chinese)

[12] Chen S D, Lan Y B, Zhou Z Y, Ouyang F, Wang G B, Huang X Y, et al. Effect of droplet size parameters on droplet deposition and drift of aerial spraying by using plant protection UAV. Agronomy, 2020; 10: 195. doi: 10.3390/agronomy10020195.

[13] Hoffmann W C, Fritz B K, Lan Y B. Using laser diffraction to measure agricultural sprays: Common sources of error when making measurements. Int J Precis Agric Aviat, 2018; 1(1): 15-18.

[14] Hassen N, Sidik N, Sheriff J. Advanced techniques for reducing spray losses in agrochemical application system. Life Science Journal, 2014; 11(3): 56-66.

[15] Caldwell D. Quantification of spray drift from aerial applications of pesticide. Master dissertation. Saskatoon: University of Saskatchewan, 2007; 106p.

[16] Baetens K, Nuyttens D, Verboven P, Schampheleire M, Nicolai R H. Predicting drift from field spraying by means of a 3D computational fluid dynamics model. Computers \& Electronics in Agriculture, 2007; 56(2): 161-173.

[17] Ru Y, Zhu C Y, Bao R. Spray drift model of droplets and analysis of influencing factors based on wind tunnel. Transactions of the CSAM, 2014; 45(10): 66-72. (in Chinese)

[18] Bird S L, Esterly D M, Perry S G. Off-target deposition of pesticides from agricultural aerial spray applications. Journal of Environmental Quality, 1996; 25(5): 1095-1104.

[19] Smith D B, Bode L E, Gerard P D. Predicting ground boom spray drift. Transactions of the ASAE, 2000; 43(3): 547-553.

[20] Liu X J, Zhou H P, Zheng J Q. Research advances of the technologies for spray drift control of pesticide application. Transactions of the CSAE, 2005; 21(1): 186-190. (in Chinese)

[21] Ross M, Carole A L. Applied weed science. Minneapolis: Burgess Publishing Company, 1985; 452p.

[22] Hobson P A, Miller P C H, Walklate P J, Tuck C R, Western N M. Spray drift from hydraulic spray nozzles: The use of a computer simulation model to examine factors influencing drift. Journal of Agricultural Engineering Research, 1993; 54(4): 293-305.

[23] Wolf R. Drift-reducing stategies and practices for ground application. technology \& health care official. Journal of the European Society for Engineering \& Mdicine, 2013; 19(1): 1-20.

[24] Yates W E, Akesson N B, Coutts H H. Drift hazards related to ultra-low-volume and diluted sprays applied by agricultural aircraft. Transactions of the ASAE, 1967; 9(6): 628-632.

[25] Yates W E, Akesson N B, Cowden R. Criteria for minimizing drift residues on crops downwind from aerial applications (of pesticide sprays on alfalfa and sugarbeets). Transactions of the ASAE, 1974; 17(4): $627-632$.

[26] Hewitt A J. Droplet size spectra classification categories in aerial application scenarios. Crop Protection, 2008; 27(9): 1284-1288.

[27] Thistle H W. Meteorological concepts in the drift of pesticides. In: Proceedings of the International Conference on Pesticide Application for Drift Management, Hawaii, 2005; pp.156-162.

[28] Grover R, Maybank J, Caldwell B C, Wolf T M. Airborne off-target losses and deposition characteristics from a self-propelled, high speed and high clearance ground sprayer. Canadian Journal of Plant Science, 1997; 77(3): 493-500.

[29] Thistle H W, Teske M E, Reardon R C. Weather effects on drift meteorological factors and spray drift: An overview. In: Proceedings of the North American Conference on Pesticide Spray Drift Management, Portland Maine, 1998; pp.64-74.

[30] Wang L, Lan Y B, Hoffmann W C, Fritz B K, Chen D, Wang S M. Design of variable spraying system and influencing factors on droplets deposition of small UAV. Transactions of the CSAM, 2016; 47(1): 15-22. (in Chinese)

[31] Wang X N. Study on spray drift and anti-drift method. Doctoral dissertation. Beijing: China Agricultural University, 2017; 120p. (in Chinese) 
[32] Zhang S C, Xue X Y, Qin W C, Sun Z, Ding S M, Zhou L X. Simulation and experimental verification of aerial spraying drift on N-3 unmanned spraying helicopter. Transactions of the CSAE, 2015; 31(3): 87-93. (in Chinese)

[33] Luo T Q, Wang Z, Yang S T, Gao L R. Numerical model of electrostatic field in electrostatic charged spray. Transactions of the CSAE, 1994; 10(4): 91-95. (in Chinese)

[34] Luo Y, Miller D R, Yang X, Mcmanus M L, Krider H M. Characteristics of evaporation from water-based bacterial pesticide droplets. Transactions of the ASAE, 1994; 37(5): 1473-1479. (in Chinese)

[35] Ranz W E, Marchall W R. Evaporation from drops. Chemical Engineering Progress, 1952; 48(173): 141-146.

[36] William R, Threadgill E. Simulation for dynamics of evaporating spray droplets in agricultural spraying. Transactions of the ASAE, 1974; 17(2): 254-261.

[37] Picot J J, Chitrangad B, Henderson G. Evaporation rate correlation for atomized droplets (for proper aerial application of insecticide sprays). Transactions of the ASAE, 1981; 24(3): 552-554.

[38] Miller P C H, Ellis M C B. Effects of formulation on spray nozzle performance for applications from ground-based boom sprayers. Crop Protection, 2000; 19(8-10): 609-615.

[39] Yates W E, Akesson N B, Coutts H H. Evaluation of drift residues from aerial applications. Transactions of the ASAE, 1966; 9(3): 389-393.

[40] Miller D R, Stoughton T E. Response of spray drift from aerial applications at a forest edge to atmospheric stability. Agricultural \& Forest Meteorology, 2000; 100(1): 49-58

[41] Miller D R, Stoughton T E, Steinke W E, Huddleston E W, Ross J B. Atmospheric stability effects on pesticide drift from an irrigated orchard. Transactions of the ASAE, 2000; 43(5): 1057-1066.

[42] Fritz K B. Meteorological effects on deposition and drift of aerially applied sprays. Transactions of the ASABE, 2006; 49(5): 1295-1301.

[43] Hoffmann W C, Salyani M. Spray deposition on citrus canopies under different meteorological conditions. Transactions of the ASAE, 1996; 39(1): 17-22.

[44] Spillman J. Atomizers for the aerial application of herbicides-ideal and available. Crop Protection, 1982; 1(4): 473-482.

[45] Dorr G J, Hewitt A J, Adkins S W, Hanan J, Zhang H C, Noller B. A comparison of initial spray characteristics produced by agricultural nozzles. Crop Protection, 2013; 53(11): 109-117.

[46] Nuyttens D, Schampheleire M D, Steurbaut W, Baetens K, Verboven P, Nicolai B, et al. Characterization of agricultural sprays using laser techniques. Aspects of Applied Biology, International advances in pesticide application, 2006; 77: 1-8.

[47] Bouse L F. Effect of nozzle type and operation on spray droplet size. Transactions of the ASAE, 1994; 37(5): 1389-1400.

[48] Miller P C H, Tuck C R, Murphy S, Ferreira M C. Measurements of the droplet velocities in sprays produced by different designs of agricultural spray nozzle. In: The 22nd European Conference on Liquid Atomization and Spray Systems, Como Lake, Italy. 2008; Paper ID: 08-8-5.

[49] Chen S D, Lan Y B, Li J Y, Zhou Z Y, Liu A M, Xu X J. Comparison of the pesticide effects of aerial and artificial spray applications for rice. Journal of South China Agricultural University, 2017; 38(4): 103-109. (in Chinese)

[50] Zhu H, Jiang Y, Li H Z, Li J X, Zhang H H. Effects of application parameters on spray characteristics of multi-rotor UAV. Int J Precis Agric Aviat, 2019; 2(1): 18-25.

[51] Kirk I W. Measurement and prediction of helicopter spray nozzle atomization. Transactions of the ASAE, 2002; 45(1): 27-37.

[52] Jong A, Michielsen J M, Stallinga H, Zande J. Effect of sprayer boom height on spray drift. In: The 52nd International Symposium on Crop Protection, Gent, Belgium, 2000; pp.919-930.

[53] Nuyttens D, Schampheleire M D, Baetens K, Sonck B. The influence of operator-controlled variables on spray drift from field crop sprayers. Transactions of the ASABE, 2007; 50(4): 1129-1140.

[54] Miller P C H, Butler E M C, Lane A G, Sullivan C M, Tuck C R. Methods for minimising drift and off-target exposure from boom sprayer applications. Aspects of Applied Biology, 2011; 106: 281-288.

[55] Miller P C H, Lane A G, Sullivan C M. Factors influencing the risk of spray drift from nozzles operating on a boom sprayer. Association of Applied Biologists, 2008; 84(5): 9-16.

[56] Tang Q, Chen L P, Zhang R R, Zhang B, Yi T C, Xu M, et al. Atomization characteristics of normal flat fan nozzle and air induction nozzle under high speed airflow conditions. Transactions of the CSAE, 2016; 32(22): 121-128. (in Chinese)

[57] Huang Y B, Plamondon C M, Thomson S J, Reddy K N. Characterizing downwind deposition of the off-target drift from aerially applied glyphosate using $\mathrm{RbCl}$ as tracer. Int J Agric \& Biol Eng, 2017; 10(3): 31-36.

[58] Chen S D, Lan Y B, Li J Y, Zhou Z Y, Jin J, Liu A M. Effect of spray parameters of small unmanned helicopter on distribution regularity of droplet deposition in hybrid rice canopy. Transactions of the CSAE, 2016 32(17): 40-46. (in Chinese)

[59] Xue X Y, Tu K, Qin W C, Lan Y B, Zhang H H. Drift and deposition of ultra-low altitude and low volume application in paddy field. Int J Agric \& Biol Eng, 2013; 7(4): 23-28.

[60] Akesson N B, Steinke W E, Yates W E. Spray atomization characteristics as a function of pesticide formulations and atomizer design. Journal of Environmental Science \& Health Part B, 1994; 29(4): 785-814.

[61] Lan Y B, Hoffmann W C, Fritz B K, Martin D E, Lopez J D. Spray drift mitigation with spray mix adjuvants. Applied Engineering in Agriculture, 2008; 24(1): 5-10.

[62] Zhang Y L, Lan Y B, Fritz B K, Xue X Y. Development of aerial electrostatic spraying systems in the United States and applications in China. Transactions of the CSAE, 2016; 32(10): 1-7. (in Chinese)

[63] Liu W L, Zhou Z Y, Chen S D. Status of aerial electrostatic spraying technology and its application in plant protection UAV. Journal of Agricultural Mechanization Research, 2018; 5: 1-9. (in Chinese)

[64] Lan Y B, Zhang H Y, Wen S, Li S H. Analysis and experiment on atomization characteristics and spray deposition of electrostatic nozzle. Transactions of the CSAM, 2018; 49(4): 130-139. (in Chinese)

[65] Carlton J B, Isler D A. Development of a device to charge aerial sprays electrostatically. Agricultural Aviation, 1966; 8(2): 44-51.

[66] Carlton J B. Electrical capacitance determination and some implications for an electrostatic spray-charging aircraft. Transactions of the ASAE, 1975; 18(4): 641-644.

[67] Carlton J B, Bouse L F, Kirk I W. Electrostatic charging of aerial spray over cotton. Transactions of the ASAE, 1995; 38(6): 1641-1645.

[68] Carlton J B, Bouse L F. Spray deposit sampling technique to evalute electrostatic aerial spray-charging. Transactions of the ASABE, 1978; 21(1): 1-5.

[69] Inculet I I, Fischer J K. Electrostatic aerial spraying. IEEE Transactions on Industry Applications, 1989; 25(3): 558-562.

[70] Kirk I W. Electrostatic coalescence for aerial spray drift mititgation. National Cotton Council of America. Beltwide Cotton Conference, Nashville, Tennessee, 2003; pp.189-191

[71] Ru Y, Zhou H P, Jia Z C, Wu X W, Fan Q N. Design and application of electrostatic spraying system. Journal of Nanjing Forestry University (Natural Science Edition), 2011; 35(1): 91-94. (in Chinese)

[72] Ru Y, Jin L, Jia Z C, Bao R, Qian X D. Design and experiment on electrostatic spraying system for unmanned aerial vehicle. Transactions of the CSAE, 2015; 31(8): 42-47. (in Chinese)

[73] Hillocks R. Farming with fewer pesticides: EU pesticide review and resulting challenges for UK agriculture. Crop Protection, 2012; 31(1): 85-93.

[74] Butler E M C, Tuck C R, Miller P C H. The effect of some adjuvants on sprays produced by agricultural flat fan nozzles. Crop Protection, 1997; 16(1): 41-50.

[75] Dexter R W. The effect of fluid properties on the spray quality from a flat fan nozzle. ASTM International, 2001; 20: 27-43.

[76] Wang X N, He X K, Song J L, Herbst A. Effect of adjuvant types and concentration on spray drift potential of different nozzles. Transactions of the CSAE, 2015; 31(22): 49-55. (in Chinese)

[77] Fan X B, Deng W, Wu G F. Research progress of spray drift control technology. Journal of Agricultural Mechanization Research, 2016; 38(6): 1-9. (in Chinese)

[78] Nordbo E. The effect of air assistance and spray quality (drop size) on the availability, uniformity and deposition of spray on contrasting targets. Bcpc Monograph, 1991; 46: 113-124.

[79] He X K, Zeng A J, He J. Effect of wind velocity from orchard sprayer on droplet deposit and distribution. Transactions of the CSAE, 2002; 18(4): 75-77. (in Chinese)

[80] Song J L, Liu Y J, Zhang J, He X K, Zeng A J, Herbst A. Drift mechanism of flat fan nozzle. Transactions of the CSAM, 2011; 42(6): 63-69. (in Chinese) 
[81] Qiu B J, Yan R, Ma J, Guan X P, Ou M X. Research progress analysis of variable rate sprayer technology. Transactions of the CSAM, 2015; 46(3): 59-72. (in Chinese)

[82] Xue X Y, Lan Y B. Agricultural aviation applications in USA. Transactions of the CSAM, 2013; 44(5): 194-201. (in Chinese)

[83] Yao W X, Lan Y B, Wen S, Zhang H H, Zhang Y L, Wang J, et al. Evaluation of droplet deposition and effect of variable-rate application by a manned helicopter with AG-NAV Guía system. Int J Agric \& Biol Eng,
2019; 12(1): 172-178.

[84] Thomson S J, Smith L A, Hanks J E. Evaluation of application accuracy and performance of a hydraulically operated variable-rate aerial application system. Transactions of the ASABE, 2009; 52(3): 715-722.

[85] Wang L H, Gan H M, Yue X J, Lan Y B, Wang J, Liu, Y X, et al. Design of a precision spraying control system with unmanned aerial vehicle based on image recognition. Journal of South China Agricultural University, 2016; 37(6): 23-30. (in Chinese) 